\title{
Primary angle-closure glaucoma, a rare but severe complication after blepharoplasty: Case report and review of the literature
}

\author{
Isabelle Francisca Petronella Maria Kappen ${ }^{1}$, Duy Thuan Nguyen ${ }^{1}$, Albert $\operatorname{Vos}^{2}$, \\ Hermanus Wilhelmus Hendricus Joseph van Tits ${ }^{1}$ \\ Departments of ${ }^{1}$ Plastic Surgery and ${ }^{2}$ Ophthalmology, Maxima Medical Center, Veldhoven, the Netherlands
}

\begin{abstract}
Blepharoplasty is one of the most commonly performed aesthetic procedures. Surgical complications are rare, but can have severe consequences, such as permanent vision loss. In this report, we describe a patient who developed primary angle-closure glaucoma (ACG) with associated vision loss after a oculoplastic procedure using local anesthesia. So far, six similar cases have been described in the literature. It is believed that acute ACG is triggered by the surgical procedure in patients with predisposing risk factors such as a cataract. Surgical triggering factors include the use of buffered lidocaine/xylocaine with adrenaline/epinephrine, stress, and coverage of the eyes postoperatively. Due to postoperative analgesic use, the clinical presentation can be mild and atypical, leading to a significant diagnostic delay. Acute ACG should therefore be excluded in each patient with postoperative complaints by assessing pupillary reactions. If a fixed mid-wide pupil is observed in an ophthalmologic examination, an immediate ophthalmology referral is warranted. Surgeons should be aware of this rare complication in order to offer treatment at an early stage and to minimize the chance of irreversible vision loss.
\end{abstract}

Keywords Glaucoma / Blepharoplasty / Postoperative complications / Oculoplasty / Blindness

\author{
Correspondence: Isabelle Francisca \\ Petronella Maria Kappen \\ Department of Plastic Surgery, \\ Maxima Medical Center, Dijk 30, 5521 \\ AZ Eersel, the Netherlands \\ Tel: $+31-612438065$ \\ Fax: +31-40-8888595 \\ E-mail: isabellekappen@me.com
}

The work was supported by the Department of Plastic Surgery of the Maxima Medical Center, Veldhoven, but required no additional funding.

Received: 30 Jun 2017 • Revised: 11 Sep 2017 • Accepted: 19 Sep 2017

pISSN: 2234-6163 • elSSN: 2234-6171 • https://doi.org/10.5999/aps.2017.01179 • Arch Plast Surg 2018;45:384-387

\section{INTRODUCTION}

Blepharoplasty is one of the most frequently performed procedures in aesthetic surgery [1]. In general, this procedure is performed under local anesthesia by infiltration of a local anesthetic and a vasoconstrictor such as adrenaline. Surgical complications are rare, but may have severe consequences, such as permanent vision loss $(0.04 \%)$, caused by retrobulbar bleeding, optical nerve ischemia, or glaucoma [2]. Early recognition and adequate differentiation among these complications are pivotal for preventing permanent vision loss.

The present report describes the development of acute angleclosure glaucoma (ACG) after blepharoplasty and ptosis repair under local anesthesia. To date, this complication has been underreported, both in the literature and in practice. The aims of this case report and review are to foster awareness of this complication; to provide insights into the diagnosis, atypical presentation, and risk factors of this condition; and to recommend measures to be taken postoperatively. 


\section{CASE}

We conducted this study in compliance with the principles of the Declaration of Helsinki.

A 64-year-old woman underwent a bilateral blepharoplasty of the upper eyelids combined with bilateral ptosis repair. The preoperative ophthalmologic examination showed no abnormalities. Visual acuity was $0.7(20 / 30)$ and $0.6(20 / 35)$ in the right and left eye, respectively. The intraocular pressures were within normal ranges, at $17 \mathrm{~mm} \mathrm{Hg}$ and $18 \mathrm{~mm} \mathrm{Hg}$ in the left and right eye, respectively.

The procedure was performed under local anesthesia by infiltrating 1\% xylocaine (lidocaine $\mathrm{HCl}$; Aspen Pharma Trading Limited, Aspen Netherlands B.V., Oss, the Netherlands) and 1:200,000 adrenaline with sodium bicarbonate. In addition, $0.04 \%$ topical oxybuprocaine $(4 \mathrm{mg} / \mathrm{mL}$; Bausch and Lomb OPS B.V., Schiphol-Rijk, the Netherlands) drops were administered. Postoperatively, the upper eyelids were cooled for $20 \mathrm{~min}$ utes every hour. The patient was discharged the same day. Hypromellose drops ( $0.3 \%$ hypromellose drops; several manufacturers) and carbomer eye gel ( $2 \mathrm{mg} / \mathrm{g}$; Bausch and Lomb OPS B.V.) were prescribed and the patient was advised to take oral analgesics as needed.

Two days after the procedure, the patient presented to the Maxima Medical Centre (the Netherlands) with blurred vision preceded by a sudden sting in the right eye and a subsequent persisting unilateral headache on the right side. Upon clinical examination, irritated conjunctivae and redness of the right eye were observed. Pupil reactions were not tested due to the mild presentation. Conjunctivitis was suspected, so Tobradex ointment $(1 \mathrm{mg} / \mathrm{g}$ of dexamethasone, $3 \mathrm{mg} / \mathrm{g}$ of tobramycin; Alcon Nederland B.V., Breda, the Netherlands) and oral analgesics were prescribed. Two days later, the periocular pain had decreased, but the patient's vision had deteriorated to the sole ability of light and dark discrimination. Upon an ophthalmologic examination conducted by an ophthalmologist, corneal edema and a fixed dilated pupil of the right eye were observed. Visual acuity was 0.01 on the right side. The intraocular pressure of the right eye had increased to $55 \mathrm{~mm} \mathrm{Hg}$ and a shallow anterior chamber was seen. Acute ACG of the right eye was diagnosed. The glaucoma was immediately treated pharmacologically, followed by an iridectomy 3 days later.

Upon follow-up 2 weeks later, the patient's vision in the right eye was still poor. In the following weeks, an iridectomy was also performed in the left eye due to a shallow anterior chamber angle, in order to prevent future acute ACG. At a 1-year followup examination, the patient's eyesight had been regained.

\section{DISCUSSION}

The development of primary ACG is a rare, but severe, complication after oculoplastic surgery that has been underreported both in the literature and in practice. Surgeons should nonetheless be aware of this possible complication, as early recognition allows for early treatment and minimizes the chance of permanent vision loss [3].

In primary ACG, there is an inadequate egress of the aqueous humor caused by blockage of the drainage system, which is made up by the trabecular meshwork situated in the anterior chamber angle. In case of a narrow anterior chamber angle, pupil dilation might result in crowding and obstruction of the angle, leading to a buildup of ocular pressure and eventually to retinal and optic nerve neuronal damage. Clinical signs and symptoms include ocular or periocular pain, nausea, a history of seeing halos around light sources, and, eventually, the development of blurred or reduced vision. Upon an ophthalmologic examination, corneal edema or a fixed mid-wide pupil unreactive to light can be observed, and both these symptoms are pathognomonic for acute ACG.

Development of glaucoma after oculoplastic surgery under local anesthesia has been previously reported. So far, six cases have been described, including one case of bilateral glaucoma (Table 1) [4-8]. In the current case, the atypical presentation of ACG contributed to a delay in the diagnosis. Similarly, clinical symptoms in the previous case reports were atypical and relatively mild, consisting mainly of blurred vision and periocular pain or headaches. Most cases did not present immediately after surgery, but between the first and fifth postoperative day. A similar delay has been noted in patients who develop bilateral acute ACG triggered by general anesthesia $[9,10]$. The use of potent perioperative analgesics or local anesthesia might attenuate the initial symptoms, leading to a milder presentation in the clinic [9]. Furthermore, an intermittent subacute form of glaucoma could precede the complete anterior chamber closure. In this subacute form, the anterior chamber angle is not completely blocked; instead, the blockage is intermittent [11]. Several portions may eventually remain closed, leading to the development of irreversible, complete ACG [11]. These patients often present with mild intermittent symptoms, such as unilateral headaches. If a patient presents with mild periocular pain, headaches, or blurred vision in the days after an oculoplastic procedure, pupil reactions should be evaluated. If a mid-wide pupil, irresponsive to light, is observed, ACG should be suspected and an acute referral to an ophthalmologist is warranted in order to prevent permanent vision loss.

Several hypotheses explaining the pathological mechanism of 


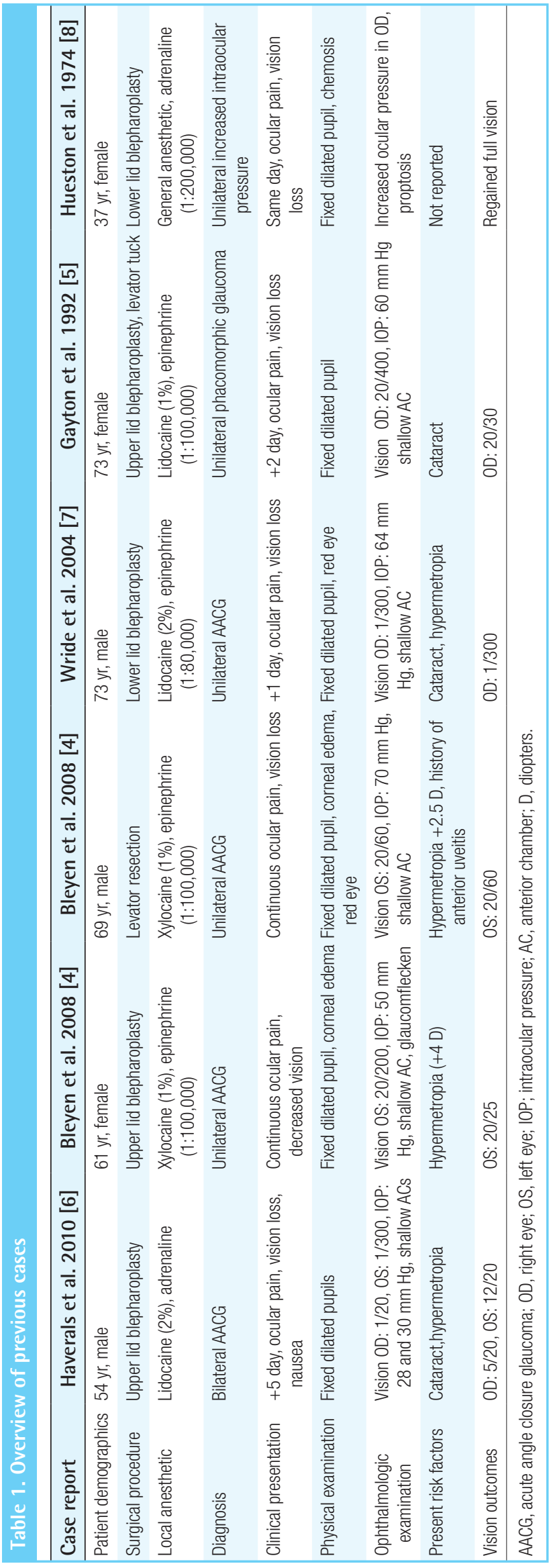

ACG after blepharoplasty have been put forward [4-7]. All propose that primary ACG is triggered in patients with predisposing risk factors causing a narrow anterior chamber angle $[7,12]$, including increased lens thickness (cataract), a relatively anteriorly positioned lens, plateau iris, advanced age, Asian ethnicity [12], and significant hyperopia. Surgical trigger factors might provoke pupil dilatation, such as stress [9], postoperative coverage of the eyes, and more importantly, pharmacologically induced mydriasis [9]. As both adrenaline and epinephrine are direct alpha-adrenergic agonists, diffusion of the anesthetic substance may result in pupil dilation. Previously, Rayatt and Khanna [13] described a case in which temporary unilateral mydriasis developed after infiltration of lidocaine and adrenaline into the eyelid. A similar phenomenon has also been reported after the infusion of adrenaline/epinephrine and lidocaine into the nasal mucosa near the lacrimal duct [14]. In such cases, mydriasis often lasted for 6-8 hours postoperatively [14]. In addition to adrenaline and epinephrine, topical lidocaine is known to increase the effect of mydriatic agents [15]. Its mydriatic potency is amplified with an increasing $\mathrm{pH}$, as a higher $\mathrm{pH}$ improves corneal penetration of the substance [15]. Combining lidocaine with sodium bicarbonate for local infusion renders the substance more basic, making it less painful during infusion, but possibly also increasing the chance of corneal penetration.

Preoperatively, it is important to consider minimizing the trigger factors associated with surgery in patients at risk. A preoperative ophthalmology referral should be considered in patients with extensive cataracts or a history of intermittent unilateral headaches, possibly masking an intermittent angle closure. In such cases, consideration should be given to avoiding buffering lidocaine with sodium bicarbonate or adding adrenaline to the local anesthetic.

Acute ACG is a rare, but severe, complication after blepharoplasty. Eyelid coverage and infusion of local anesthetics and vasoconstrictors such as adrenaline/epinephrine with the addition of sodium bicarbonate may precipitate the onset of glaucoma in patients at risk for this complication. In patients presenting with postoperative reduced vision or mild periocular pain, pupil reactions should be assessed as part of the standard workup. In case of a mid-wide fixed pupil, acute glaucoma should be suspected, and an immediate ophthalmology referral is warranted to prevent significant long-term vision loss.

\section{NOTES}

\section{Conflict of interest}

No potential conflict of interest relevant to this article was reported. 


\section{REFERENCES}

1. The American Society for Aesthetic Plastic Surgery. 2016 Cosmetic surgery national data bank statistics [Internet]. New York, NY: American Society for Aesthetic Plastic Surgery; c2016 [cited 2017 Jan 31]. Available at: https://www. surgery.org/sites/default/files/ASAPS-Stats2016.pdf.

2. Dutch Society for Plastic Surgery. Guideline: blepharoplasty of the upper eyelids [Internet]. Utrecht: Dutch Society for Plastic Surgery; c2014 [cited 2017 Jan 31]. Available at: https://www.nvpc.nl/uploads/stand/137141004DOCPL-Richtlijn\%20Blepharoplastiek\%20Bovenoogleden\%20 (revisie)\%20aangenomen\%20ALV\%20NVPC\%204\%20 oktober\%202014.pdf.

3. Andreatta W, Elaroud I, Nightingale P, et al. Long-term outcomes after acute primary angle closure in a white Caucasian population. BMC Ophthalmol 2015;15:108.

4. Bleyen I, Rademaker R, Wolfs RC, et al. Acute angle closure glaucoma after oculoplastic surgery. Orbit 2008;27:49-50.

5. Gayton JL, Ledford JK. Angle closure glaucoma following a combined blepharoplasty and ectropion repair. Ophthal Plast Reconstr Surg 1992;8:176-7.

6. Haverals K, Augustinus A, Hondeghem K. Bilateral acute angle-closure glaucoma after blepharoplasty. Bull Soc Belge Ophtalmol 2010:59-61.
7. Wride NK, Sanders R. Blindness from acute angle-closure glaucoma after blepharoplasty. Ophthal Plast Reconstr Surg 2004;20:476-8.

8. Hueston JT, Heinze JB. Successful early relief of blindness occurring after blepharoplasty: case report. Plast Reconstr Surg 1974;53:588-92.

9. Gayat E, Gabison E, Devys JM. Case report: bilateral angle closure glaucoma after general anesthesia. Anesth Analg 2011;112:126-8.

10. Jaroudi M, Fadi M, Farah F, et al. Glycopyrrolate induced bilateral angle closure glaucoma after cervical spine surgery. Middle East Afr J Ophthalmol 2013;20:182-4.

11. Sihota R. Classification of primary angle closure disease. Curr Opin Ophthalmol 2011;22:87-95.

12. Wright $C$, Tawfik MA, Waisbourd $M$, et al. Primary angleclosure glaucoma: an update. Acta Ophthalmol 2016;94: 217-25.

13. Rayatt S, Khanna A. Unilateral mydriasis during blepharoplasty. Br J Plast Surg 2001;54:648.

14. D’Souza MG, Hadzic A, Wider T. Unilateral mydriasis after nasal reconstruction surgery. Can J Anaesth 2000;47:111921.

15. Claesson M, Johansson M, Behndig A. Mydriasis with different preparations of topically administered lidocaine hydrochloride. J Cataract Refract Surg 2009;35:277-81. 